\title{
Application of Kalman Filter to prognostic method for estimating the RUL of a Bridge Rectifier
}

\author{
P.V.R. Sai Kiran ${ }^{\# 1}$, S.VijayaramKumar ${ }^{\# 2}$, P.S.Vijayakumar ${ }^{\# 3}$, Vikram Varakhedi ${ }^{\# 4}$, Vanam Upendranath ${ }^{\# 5}$ \\ \# STTD, National Aerospace Laboratories \\ Bangalore, India

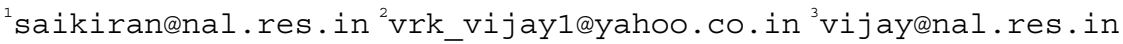 \\ "varakhedi23@gmail.com 5anam@nal.res.in
}

\begin{abstract}
Prediction of the Remaining Useful Lifetime (RUL) of the system brings down the maintenance cost, downtime and also helps to take corrective measures. This results in avoiding catastrophic events. In this Paper, The RUL prediction is done based on KalmanFilter approach with dynamic curve fitting. The desired state (which is synthetic data) of the system is estimated based on a second order Kalman Filter, where Newtonian Kinematic model is used in tracking the significant feature state of the system. The dynamic curve fitting is done based on Least Square Error Sense method. The dynamically fitted curve is extrapolated until the failure threshold is reached and subsequently RUL is estimated. The algorithm thus developed is validated by a single phase full wave bridge rectifier analogous to aircraft Transformer Rectifier Unit (TRU), to obtain the real time significant feature data. The experimental results are compared with those of developed algorithms and results show Kalman filter based algorithm is $\sim 95 \%$ accurate.
\end{abstract}

Keywords-RUL, DSP, Prognosis, Decision making, Kalman Filter.

\section{I.INTRODUCTION}

The science of Prognosis is early detection of faults or tracing of significant feature or early indications and hints of failure, wear out and fault conditions. The system or subsystem's life is predicted based on early indications so that it can be replaced or rectified in timely manner before completedegradation takes place. The extent of deviation or diversion of the critical parameters monitored forms the basis for RUL prediction of a system or a subsystem. This study of failure mode prediction is called Prognostic and Health Management (PHM) $[1, \quad 2, \quad 3, \quad$ and 4]implementationoffers a good opportunity in reducing the overalllife cyclecost and also decreases operations/maintenance cost and time [1].Complex systems like aircraft electrical systemsand avionics have a vital impact on the availability and reliability during the mission time. Technological advancements in aerospace industry are leading towards more electrical systems being integrated in aircraft [5]. Present health management in Electrical Power System (EPS) is limited to predicting impending failure [6].

The performance of the aircraft is dependent on reliability of the electrical system which is proportional to the amount of maintenance received by the system and the knowledge of those who perform such maintenance. It is therefore, important that maintenance be accomplished using the best techniques and practices to minimize the possibility of failure. Adjustments,repairs, overhaul, testing of electrical equipment and systems in accordance with the recommendations and procedures of the component manufacturer results in aircraft downtime. The time consumed by aircraft on ground can be significantly reduced by RUL estimation of its various systems. Replacement of defective/damaged electrical system LRUscan beplanned ahead byproper RUL prediction.

Kalman Filter applications include navigation,GPS and traffic management [7]. Prognosis of failure using Kalman Filtering has been demonstrated in steel bands and aircraft power generators [6].An intelligent prognostic model in the form ofalgorithm can be implementedin any system using kalman filter.

In this paper, dynamic curve fitting is implemented for RUL estimationwhich is unique from other implementations[6].This paper also addresses PHM implementation for a single phase full wave bridge rectifier experimental data for analysis of RUL estimation.The RUL estimationprocess is shown in Figure 1. 


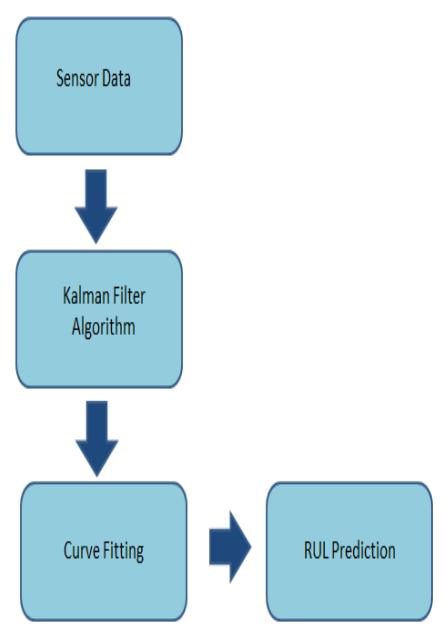

Figure 1RUL estimation process block diagram

\section{II.KALMAN FILTER}

System state estimation inthe presence of measurement noise and process noise has been achieved using Kalman Filter. Thisis regarded as an optimal solution for many tracking and data prediction tasks. It is essentially composed of a set of mathematical equations implemented asa predictor-corrector type estimator whichis optimal in the sensethat it minimizes the estimated error covariance. System state has been described in state space form using the feature vector measurementand velocity and acceleration of feature vector change [6].The state of the system at a future time instance has been computed based on the state space at preceding time step, system transition matrix, measured vector, process noise and measurement noise.

Kalman Filter equations for estimating the state with measurement noise and process noise are given by the following equations:

$$
\begin{gathered}
\hat{x}_{k}=A_{k} \hat{x}_{k-1}+K_{k}\left(Z_{k}-H_{k} A_{k} \hat{x}_{k-1}\right) \\
z_{k}=H x_{k}+v_{k}
\end{gathered}
$$

Where

$\hat{x}_{k}$-Kalman Filter estimate of the system state at $\mathrm{k}^{\text {th }}$ time step

$x_{k}-$ Actual system state at the $\mathrm{k}^{\text {th }}$ time-step

$H$ - Measurement matrix, $z_{k}$ - measurement vector

$v_{k}$-measurement noise, $\quad K_{k}$-Kalman gain

Kalman gain is calculated based on Riccati equations:

$$
\begin{gathered}
M_{k}=A_{k} P_{k-1} A_{k}^{T}+Q_{k} \\
K_{k}=\frac{M_{k} H^{T}}{H M_{k} H^{T}+R_{K}} \\
P_{k}=\left(1-K_{k} H\right) M_{k}
\end{gathered}
$$

Where

$A_{k}$ - State transition matrix,

$P_{k}$-posterior estimate of error covariance after update,

$Q_{k}$ - The process noise matrix,

$M_{k}$-Priori estimate of error covariance before update.

The significant feature characteristics used for prognosis of the system healthis takenas referencefor algorithm development. A significant feature addedwith noise, altered by faults occurred or induced in it is shown in Figure 2. The system is considered to be having four regions of operation, stating, Healthy Region (0.95 to 1.1), Caution Region (0.9 and 0.95), Repair Region (0.75 and 0.9) and Failure Region (0.75).

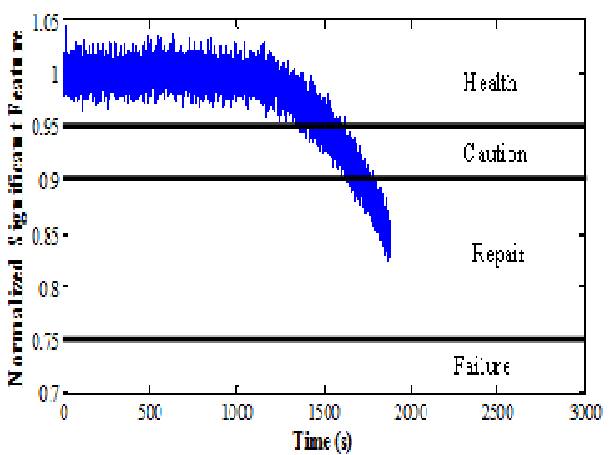

Figure 2.Significant Feature Characteristics 
Figure 2 shows that when the system is in stable condition the significant feature stays in Healthy Region, however, after the incurrence of fault the significant feature characteristics tend towards the Failure Region. Newtonian second order kinematic model used for tracking is,

$$
x_{k}=x_{k-1}+\dot{x}_{k-1} \tau+\frac{1}{2} \ddot{x}_{k-1} \tau^{2}
$$

Where, $x, \dot{x}, \ddot{x}$ are significant feature characteristics, rate of change, and acceleration of the signature feature or transfer characteristics, respectively, $\mathrm{k}$ indicates state at $\mathrm{k}^{\text {th }}$ time and $\tau$ is the time increment since the last measurement. Therefore, the state vector is defined as,

$$
X_{k}=\left[x_{k} \dot{x}_{k} \ddot{x}_{k}\right]^{T}
$$

The state vector estimate is given by equations,

$$
\begin{gathered}
\widehat{X}_{k}=A \widehat{X}_{k-1} \\
A=\left(\begin{array}{ccc}
1 & \tau & 0.5 \tau^{2} \\
0 & 1 & \tau \\
0 & 0 & 1
\end{array}\right) \\
z_{k}=H x_{k}+v_{k}
\end{gathered}
$$

A is the state transition matrix for transition of state from $\mathrm{k}-1^{\text {th }}$ step to $\mathrm{k}^{\text {th }}$ step, is derived from the Newtonian kinematic model equation (6)where $z_{k}$ the measurement at $\mathrm{k}^{\text {th }}$ time step, His the output coupling matrix and $V_{k}$ is the measurement noise. Since the position of the component significant feature characteristic is the only measured value, the measurement vector $H$ is given by,

$$
\begin{aligned}
& H=\left[\begin{array}{lll}
1 & 0 & 0
\end{array}\right]^{T}(11) \\
& z_{k}=x_{k}+v_{k}
\end{aligned}
$$

\section{II.1 Remaining Useful Lifetime (RUL) Estimation}

RUL is the useful time left on a system at a specified time of operation. Its estimation is based on Prognosis and Health Management. RUL is typically random and unknown, and it must be estimated from available sources of information obtained from in-condition to system failure.

In this paper, RUL is estimated based on curve fitting and extrapolation technique. The results obtained for the Kalman Filter tracking with respect to the measurement update is shown in Figure 3. The estimated states show the path of significant feature characteristics from Healthy to Fault conditions heading downward manner still the Time of Failure (TOF). Curve fitting and extrapolation of the significant feature are carried out after it crosses Healthy Region.

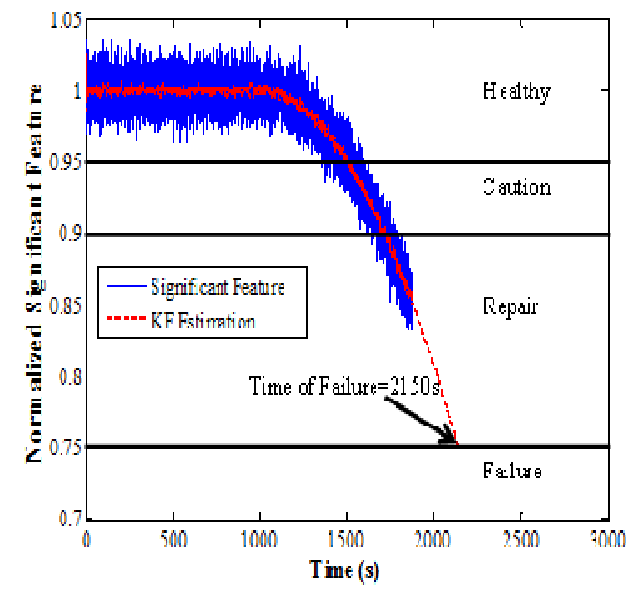

Figure 3. Tracking of Significant feature characteristics by Kalman Filter

The curve fitting technique generally referred is the Least Square Polynomial Fit. The least square error equation for least square polynomial fit is given by,

$$
e=\sum_{i}\left(x_{i}-y\right)^{2}
$$

Where, $\boldsymbol{x}_{\boldsymbol{i}}$ are the input samples and $\boldsymbol{y}$ is the curve fit of extrapolated samples. In Least Square Error Sense Fitting, the coefficients of curve fityare changed until error $(\boldsymbol{e})$ is minimal. The curve is fit at each and every step of the Kalman Filter update. The samples chosen for curve fit are present and past estimated states of the Kalman Filter. The polynomial equation used for curve fit is,

$$
y=a t^{2}+b t+c
$$

Where $t$ is time and $a, b, c$ are coefficients. The RUL of the system is calculated by extrapolating the polynomial curve until it reaches the failure threshold or it satisfies the condition given by

$$
f=a t^{2}+b t+c-0.75
$$

The time ' $\mathrm{t}$ ' is incremented until $\mathrm{f}<0$ condition is satisfied,

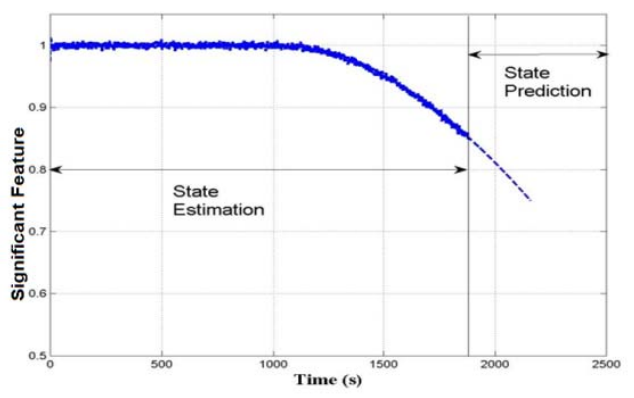

Figure 4 State estimation and prediction 
Figure 4 also shows Kalman Filter tracking, and state prediction. The prediction band considered for estimating the RUL is between $0.9-0.85$. Each and every updated state in the band is used for estimating the time of failure and RUL of the significant feature of the system. Figure 5 shows the estimated time of failure of each state within the prediction band andRUL for each state in the defined band is shown in Figure 6.

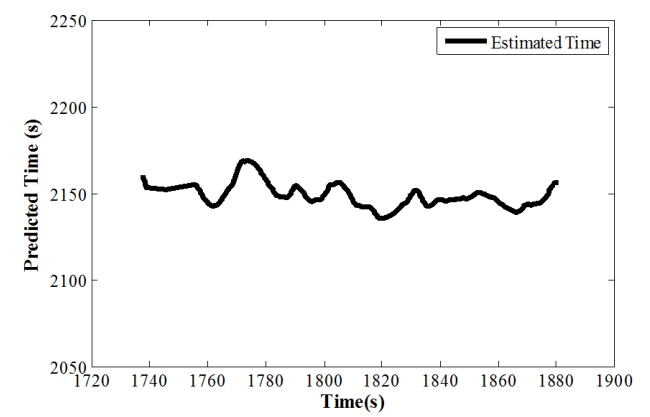

Figure 5 Estimated Time of failure in the band

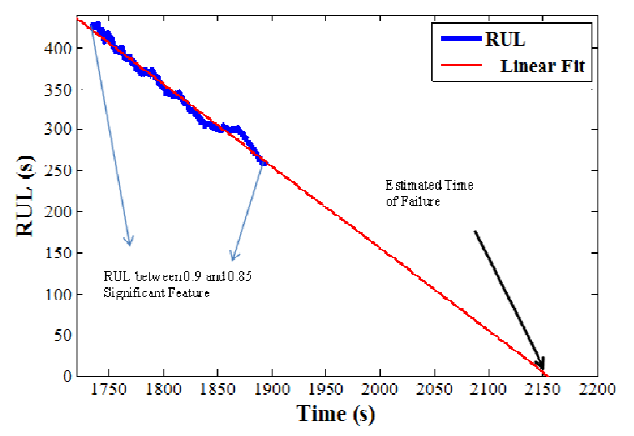

Figure 6 Estimated RULtime in the Band

In this case, exact time of failure is at $2150 \mathrm{sec}$, for each and ${ }^{\mathrm{a}}$ every state between the prediction band the time of failure is estimated subsequently from RUL is estimated around $432 \mathrm{sec}_{\mathrm{b}}$ based on TOF. The histogram representation of the estimated time of failure is shown in Figure 7.

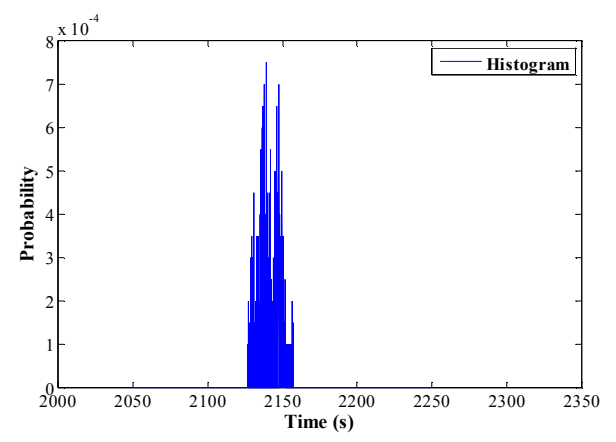

Figure 7 Histogram representation of Estimated Time of Failure

The predicted time in the histogram is varied mostly below and above the correct time of failure. The fitting of Gaussian distribution givesa better way of finding the TOF.The mean of the distribution gives the Time of failure (TOF) $2132 \mathrm{sec}$. RUL is estimated around $432 \mathrm{sec}$ based on TOF. Figure 8 shows fitting the Gaussian distribution to the TOF. Variable sample length is discussed in the following section 2.3.

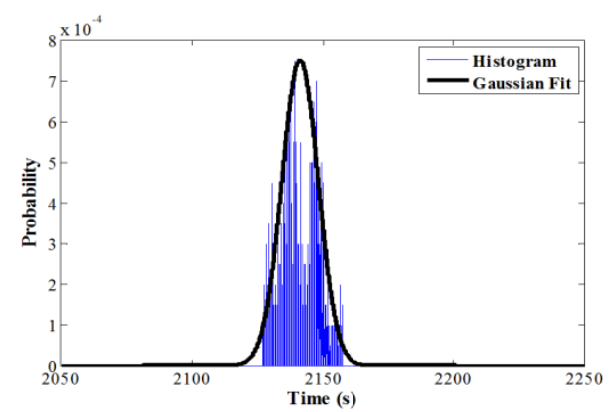

Figure 8 Gaussian fit to Estimate Time of Failure

II.2 Algorithm Steps:

1. Initialization at time $\mathrm{k}=0$,

2. State projection from $\mathrm{k}-1$ to $\mathrm{k}^{\text {th }}$ time, $\hat{x}_{k}=A \hat{x}_{k-1}$

3. Calculate error covariance matrix and Kalman Gain

$M_{k}=A_{k} P_{k-1} A_{k}^{T}+Q_{k}$

$K_{k}=\frac{M_{k} H^{T}}{H M_{k} H^{T}+R_{K}}$

4. Posterior estimate of the state with measurement

$\hat{x}_{k}=A_{k} \hat{x}_{k-1}+K_{k}\left(Z_{k}-H_{k} A_{k} \hat{x}_{k-1}\right)$

5. Update error covariance

$P_{k}=\left(1-K_{k} H\right) M_{k}$

6. Store present and past $\mathrm{N}$ states

7. If signature feature is between prediction threshold

Initialize time for prediction

$t=$ time $(\mathrm{sec}) \rightarrow$ presentstate

Curve fitting and extrapolation will be done until condition is satisfied

$t=t+1$

$f=a t^{2}+b t+c-$ failurethreshold $f<0$

Where $a, b, c$ are fitted curve coefficients

c. If $\mathrm{f}$ less than zero is not satisfied go to step 7.b

d. Computed time is the time of failure

8. Estimate Remaining Useful Lifetime defined in 2.1

9. Repeat step 2.

\section{II.3 Variable Sample Length Fitting}

The variable sample lengths are considered as 3000, 4000, 5000 and 6000 . The present and past Kalman estimated states are considered as the samples for second order curve fitting. The time of failure isestimated through the prediction technique mentioned in section 2.1.The obtained result for Gaussian fitting is shown in Figure 8. Now the sample length for fitting is increased to 4000, 5000, 6000 samples and the resulting Gaussian fit plots are shown in Figure 9. Due to this each and every curve gives different mean that is TOF values, and all curves are closer to our estimated TOF values(2132sec), RUL is estimated $432 \mathrm{sec}$ based on TOF, which is $>95 \%$ accurate. 


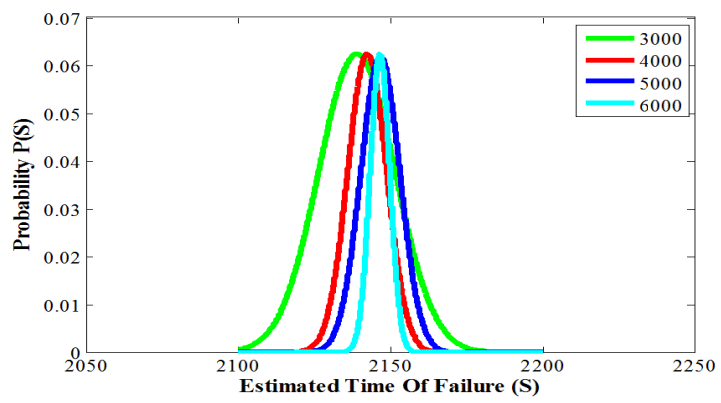

Figure 9TOF for different length fit

\section{DESCRIPTION OF EXPERIMENTAL SETUP}

To obtain the degradation data to estimate the RUL using developed algorithm, a prototype experiment setup was built using a single phase 220 volt to 24 volt step down transformer and diode bridge rectifier circuit. The experimental circuit diagram is shown in Figure 10. The function of the single phase bridge rectifier circuit is to convert the available alternating voltage into direct voltage. The DC voltage needed by the load can be obtained by rectifier circuit.

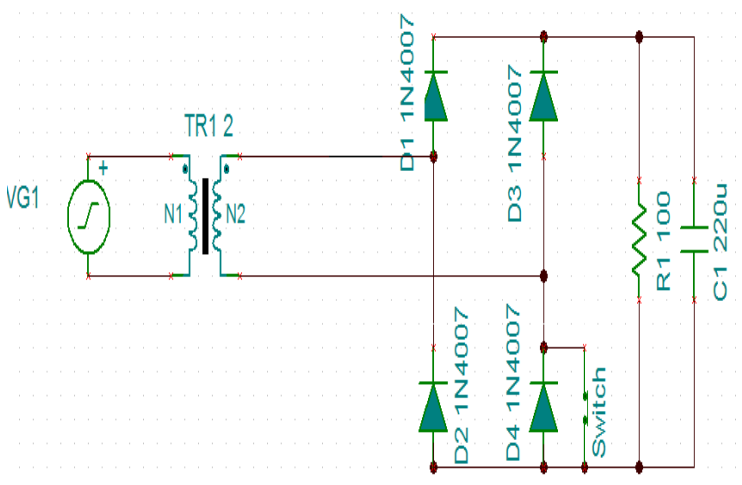

Figure 10 Bridge rectifier circuit integrated with the switch for shorting diode

First advancement of the experiment is to log the temperature data of transformer working under normal operating range. The incipient fault is injected in bridge rectifier circuit by shorting one of the four diodes by using a switch as shown in Figure 10 . The temperature change with respect to time due to harmonics is observed, before and after inserting the fault. The shorting of the diode induces harmonics. The harmonics induced at the negative half cycle of the applied input will start increasing the temperature of the transformer leading to degradation of the circuit with small variation in output voltage. The temperature of the transformer is monitored using Infrared Temperature sensor gun. The degradation triggered due to fault insertion is traced at regular interval by monitoring the change in temperature. The temperature is noted at 20 seconds interval between each temperature sample. Figure 11 shows experimental setup and the list of componentsused in Table 1.

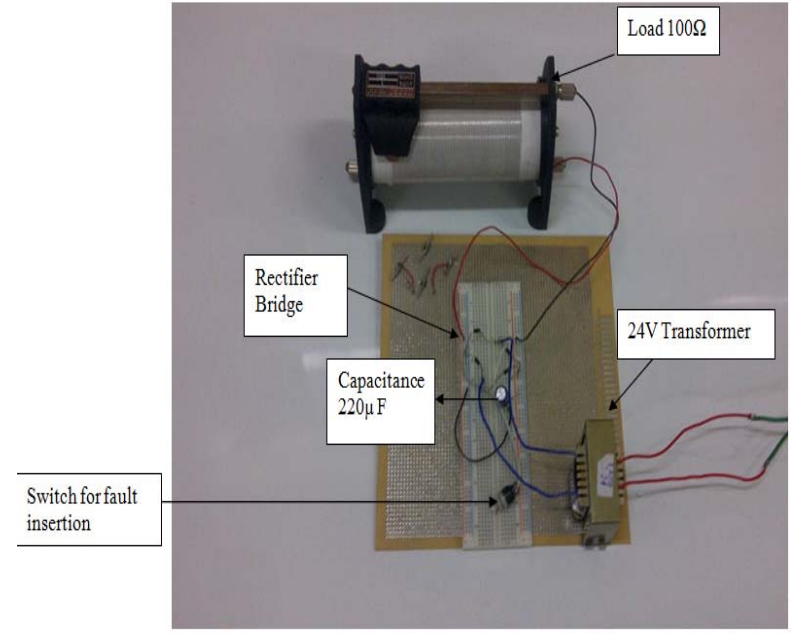

Figure 11 Schematic of experimental setup

Table 1: Experimental component specifications

\begin{tabular}{|c|c|}
\hline Components & Specification \\
\hline $\begin{array}{c}\text { Voltage source and } \\
\text { frequency }\end{array}$ & $\begin{array}{c}230 \mathrm{~V} 50 \mathrm{~Hz} \text { single phase } \\
\mathrm{AC}\end{array}$ \\
\hline Transformer & $24 \mathrm{Vrms}$ output \\
\hline Diode & $1 \mathrm{~N} 4007$ \\
\hline Load & $100 \Omega$ \\
\hline Capacitance & $220 \mu \mathrm{F}$ \\
\hline
\end{tabular}

Output voltage waveform of the full wave bridge rectifier circuit, obtained under normal working condition is shown in the Figure 12.

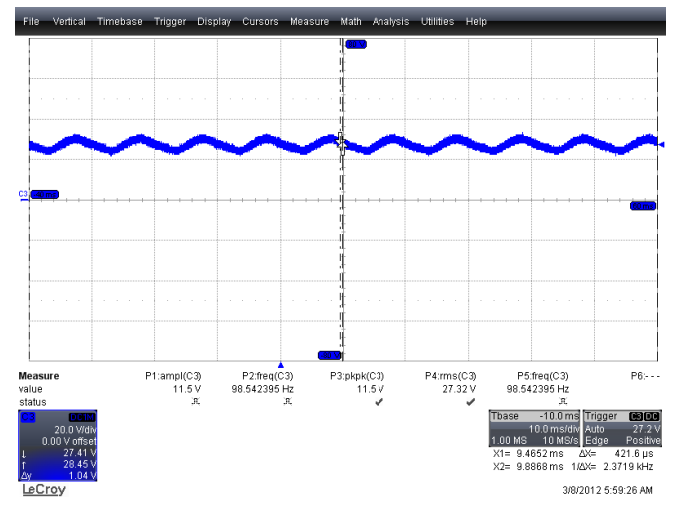

Figure 12 Output voltage without fault insertion

When the fault is inserted into the bridge rectifier circuit by shorting one of the four diodes and varied output voltage is shown in Figure 13. 


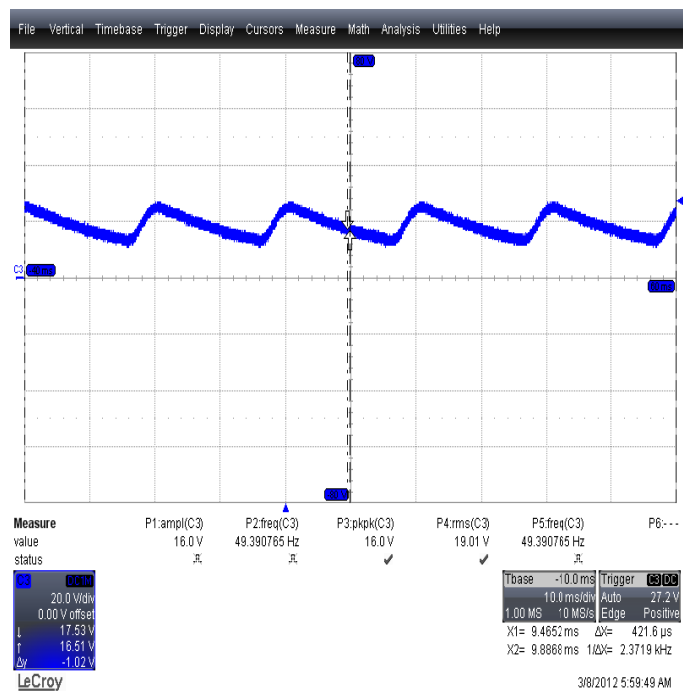

Figure 13 Output voltage with fault insertion

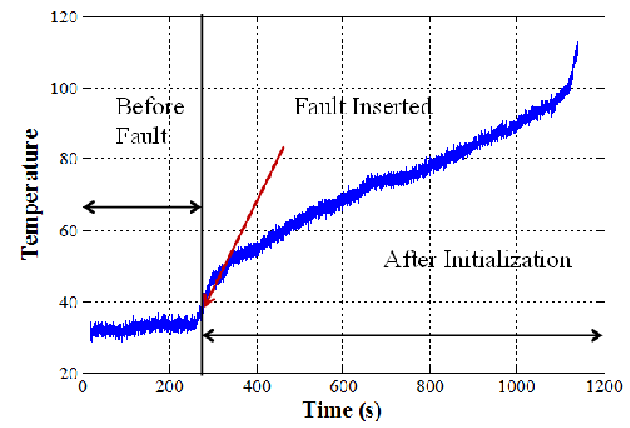

Figure 14 Plot of Temperature vs Time

Figure 14 shows a clear indication at what point the degradation is triggered and also the complete system failure by tracking the temperature parameter of the transformer. RUL can be calculated by the time difference between complete failure times to the time of fault insertion. The fault is inserted at 260 seconds, and the complete time of failure is observed at 1140 seconds as a result exact RUL is 880 seconds.

\section{VALIDATION OF EXPERIMENTAL DATA}

The temperature data, before and after fault insertion is shown in Figure 15. When the fault is injected at $260^{\text {th }}$ second the temperature started rising until transformer stopped functioning. However prior to the fault injection, temperature almost remainedconstant. This temperature change is used to track the transformer degradation. For predicting RUL, the Prognostic algorithm is implemented.

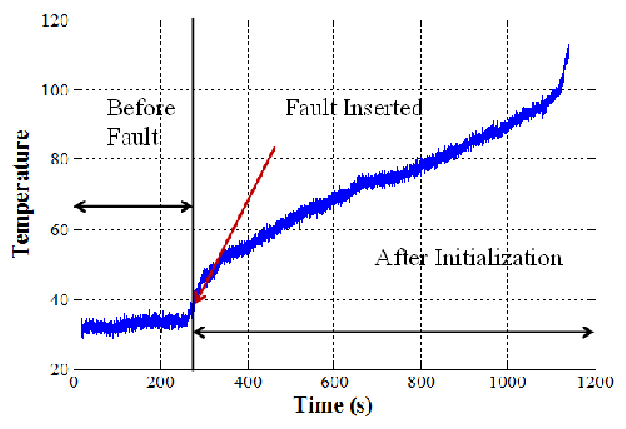

Figure 15.Temperature variation of the transformer under Healthy/faulty condition

The prediction band considered for predicting RUL is $\sim 60-70^{\circ} \mathrm{C}$. The prognostic algorithm is implemented in this band.

TOF prediction at different instances of time in the prediction band is estimated and is shown in the Figure 16. It is observed that the estimated TOF gets closer to the actualfailure time.

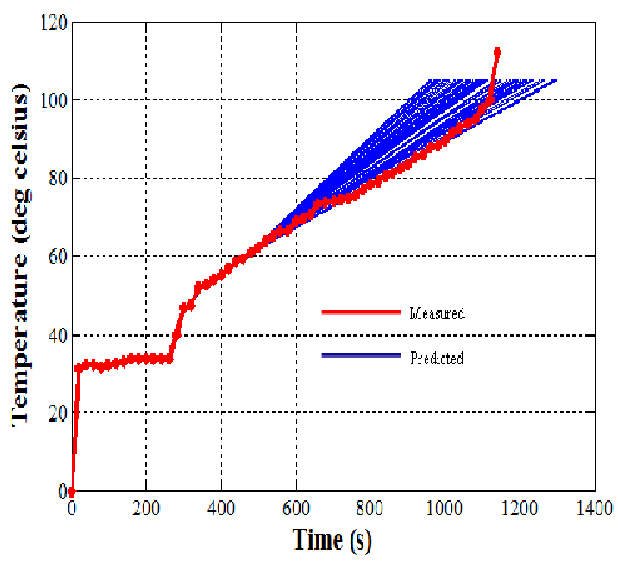

Figure 16.Measured and Predicted state of temperature

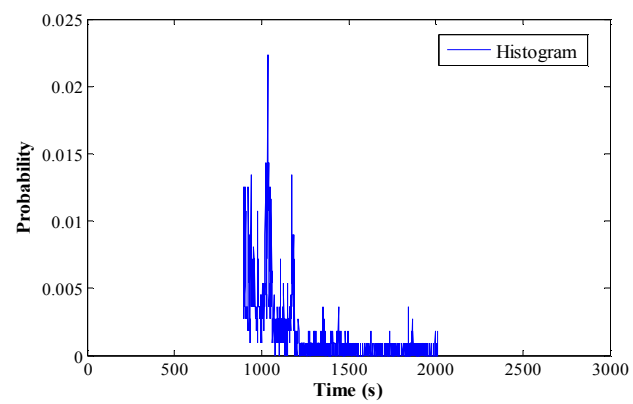

Figure 17 Probability density function(PDF) for Estimated Time of Failure(TOF).

Figure 17 shows the probability density function of the Estimated TOF. The mean value of Estimated TOF is predicted $\sim 1103 \mathrm{sec}$ and RUL estimated is $\sim 843 \mathrm{sec}$. By comparing predicted RUL with the experimentally obtained RUL which is 880 seconds the prediction shows an accuracy of $95 \%$. 
For further verification the Kalman Filter approach for RUL prediction with different sample lengthsis discussed in section II.3. Probability density function of TOF was obtained for different sample lengths of 300, 400, 500, and 600 are shown in Figure 18.

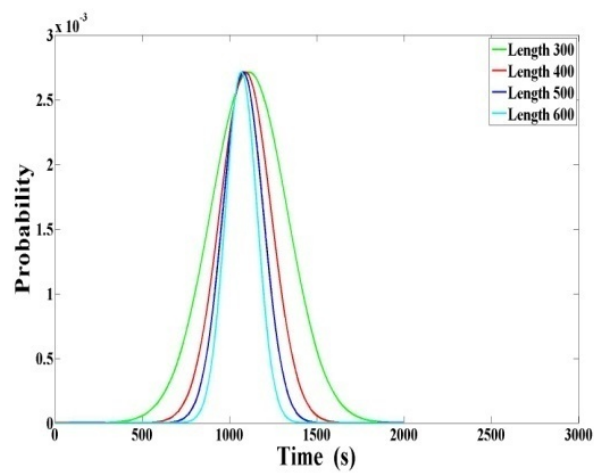

Figure 18Estimated Time of Failure (PDFs) for different length fit

From Figure 18, it is clearly understandable that, as the number of samplesare increased the standard deviation is reduced, the percentage of accuracy for every case is more than $95 \%$, with shift in mean of the gaussian distribution. The mean of the gaussian distribution indiates the TOF.

\section{CONCLUSIONS}

The Kalman Filter approach for predicting the RUL has been studied. The algorithm developed was tested for the synthetic data and the same was validated using the experimental data, the latter matches very well providing around $95 \%$ accuracy.

\section{ACKNOWLEDGEMENT}

The authors are grateful to the Dr.Satishchandra, Head, STTD, CSIR-NAL Bangalore, NPMASS and the Director, CSIR-NAL, for their generous support and encouragement. Sincere thanks are due to Dr. Kota Harinarayana, DS Kothari DRDO Chair, ADA Bangalore, for his valuable guidance, advice and help.

\section{REFERENCES}

[1]. John W. Sheppard, Mark A. Kaufman, Timothy J.Wilmer, IEEE Standards for Prognostics and HealthManagement, IEEE A\&E System Magazine, September2009

[2]. Patankar, R.; Rajagopalan, V.; Tolani, D.; Ray, A.; Begin, M.; , "Prognosis of Failure Precursor in Complex Electrical Systems Using Symbolic Dynamics," American Control Conference, 2007. ACC '07, vol., no., pp.18461851,9-13July2007 doi: 10.1109/ACC.2007.4282219

[3]. Ciandrini, C.; Gallieri, M.; Giantomassi, A.; Ippoliti, G.; Longhi, S.; , "Fault detection and prognosis methods for a monitoring system of rotating electrical machines," Industrial Electronics (ISIE), 2010 IEEE International Symposium on, vol., no., pp.2085-2090, 4-7 July 2010 doi: 10.1109/ISIE.2010.5637762

[4]. Keller, K.; Swearingen, K.; Sheahan, J.; Bailey, M.; Dunsdon, J.; Przytula, K.W.; Jordan, B.; , "Aircraft electrical power systems rognostics and

health management," Aerospace Conference, 2006 IEEE, vol., no., pp.12,doi:10.1109/AERO.2006.1656087

[5]. Downes, Clive G., ASTRAEA T7 - An architectural outline for system health management on civil UAVs, Institution of Engineering and Technology Conference on Autonomous Systems, 1-4, 2007

[6]. Batzel, T.D.; Swanson, D.C.; "Prognostic Health Management of Aircraft Power Generators," Aerospace and Electronic Systems, IEEE Transactions on, vol.45, no.2, pp.473-482, April 2009 doi: 10.1109/TAES.2009.5089535

[7]. Kalman, R., A New Approach to Linear Filtering and Prediction Problems, Transactions of the ASME--Journal of Basic Engineering, Vol. 82, No. D, pp. 35-45, 1960.

[8]. Hongwei, Bian; Zhihua, Jin; Weifeng, Tian; , "IAE-adaptive Kalman filter for INS/GPS integrated navigation system," Journal of Systems Engineering and Electronics", ,vol.17, no.3, pp.502-508, Sept. 2006 doi: 10.1016/S1004-4132(06)60086-8,

[9]. Lall, Pradeep, Lowe, Ryan, Goebel, Kai, "Prognostics using Kalman-Filter models and metrics for risk assessment in BGAs under shock and vibration loads", Electronic Components and Technology Conference (ECTC), 2010 Proceedings 60th, On page(s): $889-901$.

\section{BIOGRAPHIES}

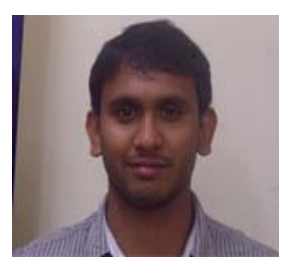

P V R Sai Kiran is currentlyworking as scientist in CSIR-NationalAerospace Laboratories(NAL), India. Hereceived his M.Tech in MachineDrives \& Power electronics from IITKharagpur and has receivedBachelor's degree in Electrical \&Electronics Engineering from JNTU. Recipient of outstanding performance award inDesign, Development and project execution for the Project of "Development of Electronics for harnessing data fromMEMS pressure sensors on LCA aircraft". His maininterests are in the areas of fault diagnosis, prognosis and Aircraft Electrical system healthmanagement.

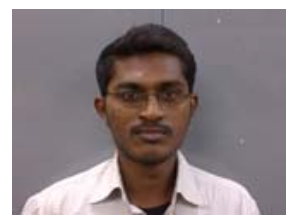

S.Vijayaramkumaris currently working as a Project Engineer in CSIR- National Aerospace Laboratories (NAL), India. Awarded Bachelor's in Electronics \& Communication Engineering in 2009.His main interests are in the areas of signal processing.

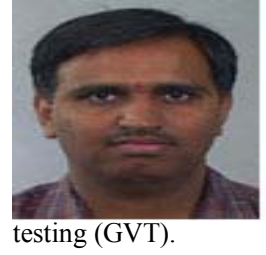

P.S.Vijayakumaris currently working as Principal scientist in CSIR-National Aerospace Laboratories (NAL), India. He received his $\mathrm{ME}$ in electronics fromVTU,Belgaum. His main interests are in the areas of signal processing, Machine/Structural fault diagnosis/Health Management and Ground Vibration

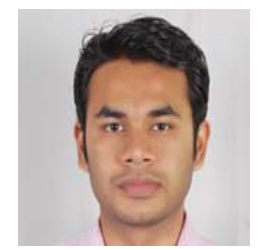

VikramVarakhedi is currently working as a Project Engineer in CSIR-National Aerospace Laboratories, India. Awarded Bachelor'sin Electronics \& Communication Engineering in 2010. CofounderElectrono Techno Solutions. Worked at National Instruments, as Technical Consultant 2011.His main interests are in the areas of automotive embedded system, $\mathrm{V} 2 \mathrm{~V}$ communication for collision avoidance. 
Dr.VanamUpendranath is a senior Principal Scientist in Structures Division at CSIR-National Aerospace Laboratories (NAL), Bangalore. $\mathrm{He}$ is the project coordinator of the NPMASS (National Program on Micro and Smart Systems) IVHM seed projects and is also incharge of the IVHM Mission initiated at CSIR-NAL. Dr.

Upendranath did his Masters in Electronics from REC

Warangal in 1981 and $\mathrm{Ph} . \mathrm{D}$ in Microelectronics from University of Trento, Italy in 2005 on Italian Govt. Fellowship. During his Ph.D. program tenure, he was also a visiting Researcher at Johns Hopkins University, Baltimore USA. Dr. Upendranath is a Senior Member of IEEE, Fellow of IETE and an elected member of Aeronautical Society of India. 
